\title{
Nuclear spin conversion of formaldehyde in protostar environments induced by non reactive collisions
}

\author{
M. Tudorie ${ }^{1}$, P. Cacciani ${ }^{1}$, J. Cosléou ${ }^{1}$, F. Herlemont ${ }^{1}$, M. Khelkhal ${ }^{1}$, C. Puzzarini ${ }^{2}$, S. Maret ${ }^{3, \star}$, and C. Kahane ${ }^{3}$ \\ ${ }^{1}$ Laboratoire de Physique des Lasers, Atomes et Molécules, UMR CNRS 8523, CERLA, Centre Lasers et Applications, \\ Université des Sciences et Technologies de Lille, 59655 Villeneuve d'Ascq Cedex, France \\ e-mail: Patrice.Cacciani@univ-lille1.fr \\ 2 Dipartimento di Chimica "G. Ciamician", Università di Bologna, via Selmi 2, 40126 Bologna, Italy \\ 3 Laboratoire d'Astrophysique de Grenoble, BP 53, 38041 Grenoble Cedex 9, France
}

Received 2 February 2006 / Accepted 7 March 2006

ABSTRACT

\begin{abstract}
Context. Formaldehyde is an important diagnostic of the physical conditions in star forming regions. The temperature of formation is determined by measuring the relative abundance of para- $\mathrm{H}_{2} \mathrm{CO}$ and ortho- $\mathrm{H}_{2} \mathrm{CO}$. The key hypothesis for this determination is that the ortho-para interconversion is strictly forbidden once the molecule is formed. However $\mathrm{H}_{2} \mathrm{CO}$ nuclear spin conversion mechanisms do exist in a gas phase either involving reactive or non-reactive collisions. This last process is governed by internal properties of the molecule, such as the mixing of energetically closed ortho-para level pairs, which are coupled through magnetic intramolecular interactions. This mixing is interrupted by a collision that makes the molecule leave the mixed state and puts it in a pure state, ortho or para, with a non zero probability for both isomeric forms.

Aims. This model allows us to estimate the spin conversion induced by non reactive collisions in different conditions encountered in the interstellar medium.

Methods. We calculated the ortho-para conversion rate in $\mathrm{H}_{2} \mathrm{CO}$ for different temperatures and $\mathrm{H}_{2}$ abundances.

Results. It is shown that the characteristic conversion time is always much longer than the $\mathrm{H}_{2} \mathrm{CO}$ lifetime.

Conclusions. Consequently, the conversion probability is zero in gas-phase protostar environments for these non reactive collisions.
\end{abstract}

Key words. molecular processes - ISM: molecules - stars: formation

\section{Introduction}

One goal of astrophysics is to determine the composition of objects (e.g. comets, stars) in the interstellar medium and to understand how they were formed. For this purpose the temperature of formation is a fundamental parameter. Its knowledge makes scenarios of formation possible. Determining the temperature of formation is made by measuring the relative abundance of ortho and para isomers for some astrophysical molecules such as $\mathrm{H}_{2}$ (Neufeld et al. 1998), $\mathrm{H}_{2} \mathrm{O}, \mathrm{H}_{2} \mathrm{CO}, \mathrm{NH}_{3}$. The key hypothesis for this determination is that the ortho-para interconversion is strictly forbidden once the molecule is formed. Thus the ortho/para ratio is conserved. It reveals the temperature at which the molecule has been formed, even though the detected signals come from a warmer gas. This is the way the temperature is being currently determined (e.g. Kahane et al. 1984; Mumma et al. 1987; Dickens \& Irvine 1999; Maret et al. 2004; Dello Russo et al. 2005; Jørgensen et al. 2005). However, nuclear spin relaxation does exist in the gas phase induced by collisions and internal properties of the molecule as recently observed for $\mathrm{CH}_{3} \mathrm{~F}$ (Cacciani et al. 2004), $\mathrm{H}_{2}{ }^{13} \mathrm{CCH}_{2}$ (Chapovsky et al. 2000), $\mathrm{H}_{2} \mathrm{CCH}_{2}$ (Sun et al. 2005), and $\mathrm{H}_{2} \mathrm{CO}$ (Peters \& Schramm 1999). The last molecule is an important diagnostic of the temperature in star forming regions (e.g. Blake et al. 1995; Jansen et al. 1994; Maret et al. 2004).

* Present address: Department of Astronomy, University of Michigan, 500 Church St., Ann Arbor MI 48109-1042, USA.
In a protostar environment, hydrogenation with $\mathrm{CO}$ molecules trapped in grain mantles is proposed as an explanation of $\mathrm{H}_{2} \mathrm{CO}$ formation (Tielens \& Allamandola 1987). Such a formation mechanism is supposed to occur at a temperature close to $10 \mathrm{~K}$. When the medium becomes warmer $(100 \mathrm{~K})$, the formaldehyde is desorbed from the mantles (Maret et al. 2004), thus going towards the gas phase where it can be detected through its rotational lines observed in emission or in absorption in the radio wavelength range. A recent experimental study demonstrated the validity of this process, revealing that the hydrogenation of $\mathrm{CO}$ produces $\mathrm{H}_{2} \mathrm{CO}$ more efficiently than do photolysis or proton bombardment under molecular cloud conditions (Watanabe \& Shiraki 2003). To be certain that this formation process is not biased by a possible nuclear spin conversion, we performed calculations by considering non reactive collisions for typical densities and temperatures in different objects of the protostar interstellar medium.

An estimation of the spin conversion rate in formaldehyde at normal conditions of pressure and temperature has already been performed by Chapovsky (2001). It is the aim of the present paper to derive the rate of the nuclear spin conversion of formaldehyde for different astrophysical gaseous environments with particular temperatures and $\mathrm{H}_{2}$ abundances for each of them (see Table 3). The characteristic conversion times are compared to other evolution times either chemical or interstellar. Newly relevant parameters have been used for such calculations.

It should be mentionned that other different mechanisms have been proposed for nuclear spin conversion involving 
reactive collisions, such as proton exchange with interstellar proton (Dalgarno et al. 1973) or the exchange of protons attached to $\mathrm{C}$ and $\mathrm{O}$ within $\mathrm{H}_{2} \mathrm{COH}^{+}$(protonation of $\mathrm{H}_{2} \mathrm{CO}$ by $\mathrm{H}_{3}^{+}$) followed by dissociative recombination (Dickens \& Irvine 1999; Ohishi et al. 1996). Such mechanisms have to be considered with the conditions of interstellar objects but are not studied in the present paper.

\section{Mechanism of the nuclear spin conversion of a molecule}

The mechanism of conversion studied here was first proposed by Curl et al. (1967): the nuclear spin might interact with the magnetic field created by the closest spins (spin-spin interaction) or by the rotation of the nuclear and electronic charges (spin-rotation interaction). The efficiency of the interactions is supposed to be enough to modify the spin. From a quantum mechanics point of view, these magnetic interactions mix energetically closed ortho and para rovibrational levels. Most of the spin modifications should funnel through these near degenerate levels.

As a possible conversion process, Miani \& Tennyson (2004) calculated the perturbation-allowed radiative ortho-to-para transitions for water. They showed that the intensity of the strongest lines is four orders of magnitude less than the intensity cut-off threshold of the usual spectrometers. Such a conversion mechanism has never been observed. Also based on Curl's hypothesis, another explanation of the conversion was developed by Chapovsky (1991). Once the molecule reaches such a pair of interacting levels, its eigenstate is a linear combination of the "pure" states corresponding to each isomer. Therefore it has a non zero probability of being projected after the next collision into the same subspace or into the other. The modification to another value of the total nuclear spin is thus possible. The quantitative description of this mechanism, called "quantum relaxation", has been developed in the framework of the density matrix formalism. It is shown that the relaxation of a nonequilibrium excess of concentration of, e.g. ortho molecules, $\delta \rho_{\mathrm{o}}(0)$ created at the instant $t=0$, will decay exponentially to zero as:

$\delta \rho_{\mathrm{o}}(t)=\delta \rho_{\mathrm{o}}(0) \mathrm{e}^{-\gamma t}$

where $\gamma$ is the conversion rate expressed as:

$\gamma=\sum_{\alpha \in \text { ortho } \alpha^{\prime} \in \text { para }} \frac{2 \Gamma_{\alpha \alpha^{\prime}}\left|V_{\alpha \alpha^{\prime}}\right|^{2}}{\Gamma_{\alpha \alpha^{\prime}}^{2}+\omega_{\alpha \alpha^{\prime}}^{2}}\left[W_{\mathrm{B}}(\alpha)+W_{\mathrm{B}}\left(\alpha^{\prime}\right)\right]$.

The summation has to be made over all ortho and para level pairs, $V_{\alpha \alpha^{\prime}}$ is the matrix element of the magnetic interaction expressed in $\hbar$ units, $W_{\mathrm{B}}(\alpha)$ is the Boltzmann factor, $\hbar \omega_{\alpha \alpha^{\prime}}$ the energy difference between the levels of the pair, and $\Gamma_{\alpha \alpha^{\prime}}$ the collisional decay rate of the coherence between the levels created by interaction $V_{\alpha \alpha^{\prime}}$. Several experimental confirmations of the validity of this model have been performed (e.g. Cacciani et al. 2004).

\section{Calculation of the spin conversion rates}

In this section we present the relevant parameters we used for the calculation of nuclear spin conversion rates $\gamma$.

\subsection{Energy difference between ortho and para states}

In an asymmetric top molecule, the levels are characterized by the total angular momentum $J$ and labelled by the $K_{\mathrm{a}}$ and $K_{\mathrm{c}}$ numbers, which refers to the projection of the angular momentum $J$ along the A and $\mathrm{C}$ inertial axis considering the prolate and oblate limiting cases. $\mathrm{In}_{2} \mathrm{CO}$, the A axis is along the $\mathrm{CO}$ bond, whereas the $\mathrm{C}$ axis is defined in the direction perpendicular to the molecular plane. The asymmetry is low and the molecule is nearly prolate. It follows that the symmetry, and consequently the spin character, of the total wavefunction of the molecule in its electronic and vibrational ground state is characterized by the parity of the $K_{\mathrm{a}}$ number: $K_{\mathrm{a}}$ is even for para formaldehyde ( $I=0$ spin wave function of $B_{1}$ symmetry), and $K_{\mathrm{a}}$ is odd for ortho formaldehyde ( $I=1$ spin wave function of $A_{1}$ symmetry). The assignment of close interacting ortho and para states was achieved after the calculation of the energy levels with the molecular parameters derived by Müller et al. (2000).

Formaldehyde belongs to the $\mathrm{C}_{2 v}$ symmetry group. Consequently, introducing the Wang basis (Landau \& Lifshitz 1981) $|\{J, K\}\rangle(0 \leq K \leq J)$ defined by the symmetric and antisymmetric combination of the functions of the symmetric top molecule $|J, K\rangle$ and $|J,-K\rangle$, the diagonalization of the Hamiltonian is reduced to the diagonalization of four independent submatrices, each of a particular symmetry $\left(A_{1}, A_{2}, B_{1}, B_{2}\right)$. The wave functions can be written as

$\left|J, K_{\mathrm{a}}, K_{\mathrm{c}}\right\rangle=\sum_{K} A_{K}|\{J, K\}\rangle$

where $A_{K}$ are expansion coefficients that also depend on $J, K_{\mathrm{a}}$, $K_{\mathrm{c}}$ quantum numbers.

Energy differences of the most effective pairs are listed in Table 1. The selection rules have been derived through the nature of the spin-rotation interactions: $\Delta J \leq 1 ; K_{\mathrm{a}}$ and $K_{\mathrm{a}}^{\prime}$ have opposite parity (ortho/para character of the pair). The fully symmetric nature of the spin-rotation operator leads to $K_{\mathrm{c}}$ and $K_{\mathrm{c}}^{\prime}$ having the same parity.

\subsection{Spin-rotation interaction}

The coupling between ortho and para states occurs via the intramolecular magnetic interactions. Symmetry considerations demonstrate that the spin-spin interaction term cannot couple ortho and para states in $\mathrm{H}_{2} \mathrm{CO}$ (Chapovsky 2001). The spin rotation interaction term is the sum of nuclear and electronic contributions. Chapovsky could only estimate the first one.

In order to have a complete and more confident value, the total $\boldsymbol{C}_{K}$, nuclear $\boldsymbol{C}_{K}^{\mathrm{nucl}}$, and electronic $\boldsymbol{C}_{K}^{\mathrm{el}}$ spin-rotation tensors were determined using ab initio computations. The electronic contribution $C_{K}^{\mathrm{el}}$ can be theoretically defined as the second derivative of the electronic energy $E^{\mathrm{el}}$ with respect to the rotational angular momentum $\boldsymbol{J}$ and the nuclear spin $\boldsymbol{I}_{K}$, whereas the nuclear contribution $C_{K}^{\text {nucl }}$ only depends on the molecular geometry. The calculations were carried out at the coupled-cluster singles and doubles (CCSD) level augmented by a perturbative treatment of triple excitations (CCSD $(\mathrm{T})$ ) (Raghavachari et al. 1989), correlating all electrons (all) and employing the (augmented) core-valence (aug)-cc-pCVnZ ( $n=\mathrm{T}$, Q) basis sets (Dunning 1989; Kendall et al. 1992; Woon \& Dunning 1995). All calculations were based on the equilibrium geometry provided by Carter \& Handy (1996), and performed with a local version of the ACES 2 program package (Stanton et al. 1992) employing perturbation-dependent basis functions (also referred to as rotational London atomic orbitals (LAOs)), in order to improve 
Table 1. Main ortho-para pairs involved in the nuclear spin conversion of formaldehyde. Conversion rates are given for a pressure of 1 Torr.

\begin{tabular}{crrrr}
\hline \hline Level pairs & $\omega / 2 \pi(\mathrm{GHz})$ & Energy $\left(\mathrm{cm}^{-1}\right)$ & \multicolumn{2}{c}{$\gamma / \mathrm{P}\left(10^{-5} \mathrm{~cm}^{-1}\right.$ Torr $\left.^{-1}\right)$} \\
$J_{\text {ortho }}, K_{\mathrm{a}}, K_{\mathrm{c}}-J_{\text {para }}, K_{\mathrm{a}}^{\prime}, K_{\mathrm{c}}^{\prime}$ & & & $T=30 \mathrm{~K}$ & $T=300 \mathrm{~K}$ \\
\hline $17,3,15-18,2,17$ & -6.16 & 445.606 & 0.00 & 155.38 \\
$9,1,8-8,2,6$ & 9.25 & 120.854 & 51.65 & 48.77 \\
$22,3,19-21,4,17$ & -10.38 & 692.111 & 0.00 & 31.85 \\
$14,1,14-13,2,12$ & 17.21 & 253.665 & 0.06 & 18.57 \\
$13,1,13-12,2,11$ & -19.31 & 221.043 & 0.20 & 14.20 \\
$14,3,11-15,2,13$ & 32.60 & 329.102 & 0.00 & 5.91 \\
$28,5,23-29,4,25$ & -11.36 & 1190.528 & 0.00 & 5.59 \\
$24,3,22-23,4,20$ & 33.05 & 802.916 & 0.00 & 2.40 \\
$23,3,21-22,4,19$ & -35.77 & 744.637 & 0.00 & 2.37 \\
$16,3,14-17,2,16$ & 51.64 & 404.251 & 0.00 & 2.28 \\
$15,1,15-14,2,13$ & 51.03 & 288.585 & 0.00 & 2.13 \\
\hline $3,1,2-4,0,4$ & -30.31 & 23.234 & 70.99 & 0.99 \\
$2,1,1-3,0,3$ & 34.62 & 15.710 & 32.46 & 0.33 \\
$4,1,3-5,0,5$ & -92.21 & 33.263 & 9.14 & 0.20 \\
$5,1,4-6,0,6$ & -150.81 & 45.793 & 3.15 & 0.12 \\
$8,1,7-7,2,5$ & -78.10 & 98.358 & 1.42 & 0.51 \\
$6,1,5-7,0,7$ & -205.80 & 60.823 & 1.26 & 0.09 \\
$1,1,0-2,0,2$ & 102.34 & 10.693 & 1.25 & 0.01 \\
Total rate (All pairs) & & & 174.88 & 309.63 \\
\hline
\end{tabular}

Table 2. Spin-rotation tensors $(\mathrm{kHz})$ of $\mathrm{H}_{2} \mathrm{CO}$.

\begin{tabular}{lrrrrrr}
\hline \hline & \multicolumn{2}{c}{ (all)CCSD(T)/aug-cc-pCVQZ } & vibrationally & Fabricant et al. & Chapovsky \\
& total & electronic & nuclear & averaged & $(1977)$ & $(2001)$ \\
\hline $\boldsymbol{C}(H)$ & & & & & & \\
$C_{a a}$ & -6.50 & +124.50 & -131.00 & -4.77 & $-3.71(16)$ & \\
$C_{b b}$ & +2.00 & +17.46 & -15.46 & +1.94 & $+2.09(4)$ & \\
$C_{c c}$ & -2.77 & +26.60 & -29.37 & -2.56 & $-2.41(22)$ & -6.78 \\
$C_{a b}$ & \pm 21.92 & $\mp 77.82$ & \pm 99.90 & \pm 21.00 & & 0.00 \\
$C_{b a}$ & \pm 3.74 & $\mp 9.91$ & \pm 13.65 & \pm 3.64 & & \\
\hline
\end{tabular}

basis-set convergence (Gauss et al. 1996). As plenty of evidence exists for the high accuracy obtained at this level of theory in the calculation of NSRCs (see for example Puzzarini et al. 2005, and references therein), we consider the $\operatorname{CCSD}(\mathrm{T})$ approach an adequate choice for the present study. Additionally, zero-point vibrational (ZPV) corrections to the spin-rotation tensors were obtained using a perturbational approach; the vibrational corrections, computed at the (all)CCSD(T)/cc-pCVTZ level, are defined as the difference between the vibrationally-averaged and the equilibrium values and added to the (all)CCSD(T)/aug-ccpCVQZ values. The results obtained are gathered in Table 2 and compared to the available experimental data.

The values of the spherical spin-rotation components $C_{ \pm 1}^{(1)}$ and $C_{ \pm 1}^{(2)}$ can be derived from the Cartesian tensor components:

$C_{ \pm 1}^{(2)}=\mp \frac{1}{2}\left(C_{a b}+C_{b a}\right) \quad C_{ \pm 1}^{(1)}=\frac{1}{2}\left(C_{a b}-C_{b a}\right)$.

The calculation is then performed according to the equations given by Chapovsky (2001). Briefly, the sum over degenerate quantum numbers ( $m, \sigma$ projection on the laboratory $Z$ axis of angular momentum and total spin of the two hydrogen atoms respectively) associated to a ortho/para pair $\psi / \psi^{\prime}$ is given by:

$$
\begin{aligned}
& \sum_{m, \sigma}\left|\left\langle\psi\left|V_{S R}\right| \psi^{\prime}\right\rangle\right|^{2}=\frac{1}{4}(2 J+1)\left(2 J^{\prime}+1\right) \\
& \times \mid \sum_{K>0, K^{\prime}>0} A_{K} A_{K^{\prime}}^{\prime} \Phi\left(J, K \mid J^{\prime}, K^{\prime}\right) \\
& +\left.\frac{1+(-1)^{J^{\prime}+K_{\mathrm{c}}}}{\sqrt{2}} A_{1} A_{0}^{\prime} \Phi\left(J, 1 \mid J^{\prime}, 0\right)\right|^{2} .
\end{aligned}
$$

The notation used in Eq. (5) refers to the calculation of matrix elements in the spherical top basis:

$$
\begin{aligned}
\Phi\left(J, K \mid J^{\prime}, K^{\prime}\right)= & \sum_{l} \sqrt{2 l+1} C_{q}^{(l)}\left(\begin{array}{ccc}
J^{\prime} & l & J \\
-K^{\prime} & q & K
\end{array}\right) \\
& \times\left[y(J)(-1)^{l}\left\{\begin{array}{rrr}
J^{\prime} & J & l \\
1 & 1 & J
\end{array}\right\}+y\left(J^{\prime}\right)\left\{\begin{array}{rrr}
J & J^{\prime} & l \\
1 & 1 & J^{\prime}
\end{array}\right\}\right],(6)
\end{aligned}
$$

where (: : :) stands for the $3 \mathrm{j}$-symbol; $\{:::\}$ stands for the $6 \mathrm{j}$-symbol; $y(J)=(J(J+1)(2 J+1))^{1 / 2}$. Parities of $K$ (ortho) and $K^{\prime}$ (para) only lead to consider $q= \pm 1$ of the spherical components of the spin-rotation $C_{q}^{(l)}$ tensor of rank $l(l=1,2)$.

\subsection{Collision-induced decoherence rate}

The spin-rotation interaction creates a coherence between ortho and para states, which is destroyed by collisions at a rate $\Gamma$. According to Eq. (2), the knowledge of $\Gamma$ is needed to calculate the conversion rate. As this value is usually unknown, the common assumption is that the coherence between two states is broken if the molecule leaves one state or the other:

$\Gamma_{\alpha, \alpha^{\prime}}=\frac{\Gamma_{\alpha}+\Gamma_{\alpha^{\prime}}}{2}$

where $\Gamma_{\alpha}$ and $\Gamma_{\alpha^{\prime}}$ are the relaxation rates of the populations $\alpha$ and $\alpha^{\prime}$, respectively. These values are commonly derived from the collisional broadening measurements of transitions involving these states. As we have shown in previous papers (Cacciani et al. 2006), results of line broadening measurements can be considered as a quantitative estimation of $\Gamma_{\alpha, \alpha^{\prime}}$. 
Table 3. Calculated spin conversion of $\mathrm{H}_{2} \mathrm{CO}$ in different interstellar media.

\begin{tabular}{|c|c|c|c|c|c|}
\hline Source & Type & Density & Temp. & Notes & Conversion time \\
\hline L1544 (1) & Prestellar core & $10^{6}$ & $5-10 \mathrm{~K}$ & $\begin{array}{l}\text { Values at } R=0.01 \mathrm{pc} \\
\text { from the center (Doty } \\
\text { et al. 2005) }\end{array}$ & $6.2 \times 10^{7}$ years \\
\hline DM-Tau (2) & $\begin{array}{l}\text { Protoplanetary } \\
\text { Disks }\end{array}$ & $10^{7}$ & $20 \mathrm{~K}$ & $\begin{array}{l}\text { Value } R=200 \text { au } \\
\text { from center and } z=0 \\
\text { (Aikawa et al. 2002) }\end{array}$ & $3.4 \times 10^{6}$ years \\
\hline Orion PDR (3) & $\begin{array}{l}\text { Photodissociation } \\
\text { region }\end{array}$ & $2 \times 10^{5}$ & $75 \mathrm{~K}$ & $\begin{array}{l}\text { (Tielens \& Hollenbach } \\
\text { 1985) }\end{array}$ & $2.8 \times 10^{7}$ years \\
\hline IRAS 16293-2422 (4) & Class 0 protostar & $3 \times 10^{8}$ & $100 \mathrm{~K}$ & $\begin{array}{l}\text { Values at } R=100 \\
\text { au from the center } \\
\text { (Schöier et al. 2002) }\end{array}$ & $1.7 \times 10^{4}$ years \\
\hline HH-54 & Outflow source & $\geq 10^{5}$ & $650 \mathrm{~K}$ & (Neufeld et al. 1998) & $1.1 \times 10^{7}$ years \\
\hline
\end{tabular}

In protostellar environments, the collisional broadening is due to collisions with $\mathrm{H}_{2}$, the main constituent. Data on broadening coefficients of $\mathrm{H}_{2} \mathrm{CO}$ with $\mathrm{H}_{2}$ in the literature helps in assigning the values of $\Gamma_{\mathrm{H}_{2} \mathrm{CO}-\mathrm{H}_{2} \mathrm{CO}}$ and, mainly, $\Gamma_{\mathrm{H}_{2} \mathrm{CO}-\mathrm{H}_{2}}$.

The self-broadening (collision $\mathrm{H}_{2} \mathrm{CO}-\mathrm{H}_{2} \mathrm{CO}$ ) depends on the moderately long-range dipole-dipole interaction $\left(\mu\left(\mathrm{H}_{2} \mathrm{CO}\right)=\right.$ 2.3321(5) D, Fabricant et al. 1977). The first relevant paper, published by Nerf (1975), presents results obtained in the millimeter range for $J=1, \cdots, 9$ at 0.5 Torr $(1$ Torr $=133 \mathrm{~Pa})$, giving a self-broadening coefficient equal to $1.57 \times 10^{8} \mathrm{~s}^{-1} \mathrm{Torr}^{-1}$. A recent study of cavity ring down spectroscopy at 50 mTorr yields a line-broadening coefficient of $1.88 \times 10^{8} \mathrm{~s}^{-1} \mathrm{Torr}^{-1}$ (Corner et al. 2003). This value was also found by Burkart \& Schramm (2003) for the broadening of the infrared $4_{14}-5_{12}$ transition in the $v_{1}$ band. We retained this value for calculating the spin conversion rate $\gamma$ in pure formaldehyde.

The $\mathrm{H}_{2}$ broadening of $\mathrm{H}_{2} \mathrm{CO}$ is dominated by a relatively strong dipole-quadrupole interaction: $Q\left(\mathrm{H}_{2}\right)=0.8 \mathrm{D} \cdot \AA$ (Nerf 1975). Nerf (1975) gave a value of $3.47 \times 10^{7} \mathrm{~s}^{-1} \mathrm{Torr}^{-1}$ for partial pressures of $\mathrm{H}_{2} \mathrm{CO}$ between 100-200 mTorr and $\mathrm{H}_{2}$ partial pressure ranging up to 900 mTorr. Burkart \& Schramm (2003) find a higher value for the $\mathrm{H}_{2} \mathrm{CO}-\mathrm{H}_{2}$ broadening coefficient, $7.68 \times 10^{7} \mathrm{~s}^{-1} \mathrm{Torr}^{-1}$, for a pressure range of $0.1-10$ Torr. As we were not able to conclude anything about the quality of these experimental values, we performed the calculations of the conversion rates in astrophysical conditions with a value of $\Gamma_{\mathrm{H}_{2}} \mathrm{CO}-\mathrm{H}_{2}$ equal to $5 \times 10^{7} \mathrm{~s}^{-1}$ Torr $^{-1}$.

All the above experimental results were obtained at room temperature. However, it is known that the line broadening increases with the inverse of temperature. This is shown by Green (1991) who calculated the state-to-state collision rates for temperatures in the range of 10 to $300 \mathrm{~K}$ (Green 1991). But these values are not helpful in our model described by Eq. (2), as the rate $\Gamma$ is defined from all transitions starting from one definite state to the others with the same ortho/para symmetry. Therefore, the temperature dependence is pertinent. In this paper the rates are calculated for transitions between states of the same spin symmetry. In our study, we used the current semi-empirical equation (Birnbaum 1967):

$\Gamma(T)=\Gamma\left(T_{0}\right)\left(\frac{T_{0}}{T}\right)^{n}$

where $T_{0}$ is the ambient temperature and $n=1$ for dipole-dipole interaction, and $n=0.8$ for the dipole-quadrupole interaction.

The broadening coefficient depends on the quantum states, but with differences of a few percent (Nerf 1975; Green 1991). Thus the linewidths are independent, within experimental errors, of the rotational $J$ quantum numbers. This was confirmed by
Niko van Hulst et al. (1987). For this reason we considered that

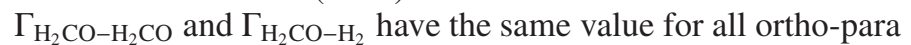
level pairs for a given temperature.

\subsection{Discussion}

The energy levels were calculated with $J \leq 50$ and $K_{\mathrm{a}} \leq 50$. All the possible ortho/para pairs were then considered for the calculation of spin conversion rates. The main contributing pairs are therefore dependent on the energy difference $\omega_{\alpha \alpha^{\prime}}$ and on the Boltzmann factor. Table 1 presents the pairs sorted by the importance of their contribution. The first five pairs, which are those contributing the most at room temperature, are given by Chapovsky (2001). In this Table 1 the relaxation rate $\Gamma$ is $1.88 \times$ $10^{8} \mathrm{~s}^{-1} /$ Torr for $T=300 \mathrm{~K}$ and $11.8 \times 10^{8} \mathrm{~s}^{-1} /$ Torr for $T=30 \mathrm{~K}$ (see Eq. (8)). The nuclear spin conversion is given for the pure gas (collisions $\mathrm{H}_{2} \mathrm{CO}-\mathrm{H}_{2} \mathrm{CO}$ ).

The importance of the pairs involving low $J$ values is crucial for the validity of the calculation at low temperature. The contribution of these pairs to the determination of $\gamma$ is significantly changed as shown in the last two columns of Table 1. Checking first the validity of calculating the nuclear spin conversion rate $\gamma_{\mathrm{H}_{2} \mathrm{CO}}$ can be performed by comparing with experimental or calculated values of the literature. Our value of gamma is $3.08 \times 10^{-3} \mathrm{~s}^{-1}$ at a pressure of one Torr and at ambient temperature, with the use of all possible ortho/para pairs. This value is three times the experimental value obtained by Peters \& Schramm (1999) and thirty times the calculated value of Chapovsky (2001). This latter discrepancy is easily explained since our spin-rotation parameter is about three times higher than Chapovsky's and it appears as a square in Eq. (2).

The temperature dependence of the characteristic time $(1 / \gamma)$ of spin conversion is given in Fig. 1. It was calculated by including the variation in the Boltzmann factor of each pair. For the low pressure regime $(\Gamma \ll \omega)$, Eq. (2) shows that $\gamma$ is proportional to $\Gamma$. Therefore the pressure dependence of the spin conversion rate $\gamma$ is linear at astrophysics pressures. The peculiar rates for astrophysical objects given in Table 3 are drawn in Fig. 1.

In the environments considered in Table 3 , the conversion times appear to be extremely long. As a comparison, the chemical equilibrium in a dense molecular cloud $\left(n\left(\mathrm{H}_{2}\right)=2 \times 10^{4} \mathrm{~cm}^{-3}\right.$, $T=10 \mathrm{~K}$ ) is typically reached after $10^{7} \mathrm{yr}$ (e.g. Markwick et al. 2000), and would be reached even faster in the denser environments considered here. In the case of the Class 0 protostar IRAS 16293-2422, the conversion time in the dense and hot part of the envelope (the so-called hot core) is shorter $\left(\sim 10^{4} \mathrm{yr}\right)$. However, this conversion time is comparable to the timescale 


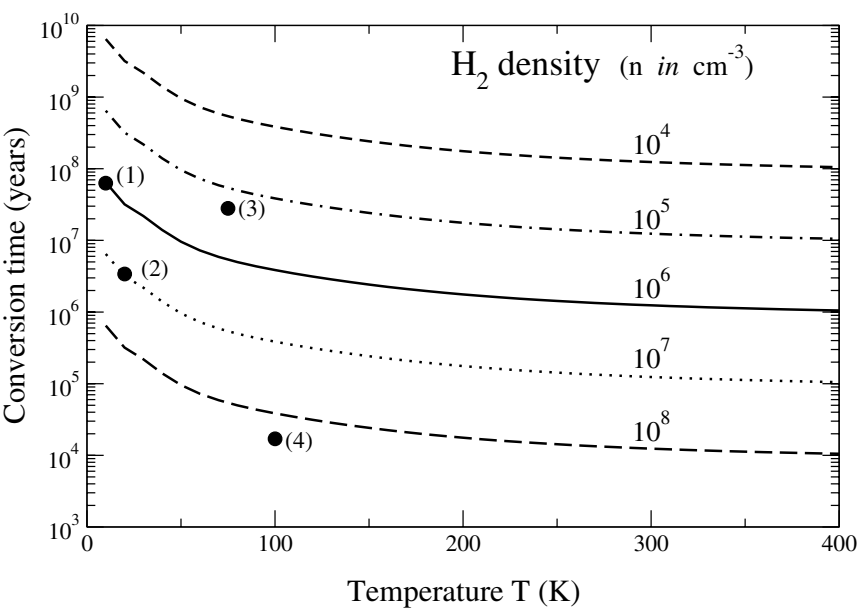

Fig. 1. Nuclear spin conversion time (in years) of $\mathrm{H}_{2} \mathrm{CO}$ induced by collisions with $\mathrm{H}_{2}$. Numbers in parenthesis refer to the different interstellar objects described in Table 3.

for the destruction of formaldehyde by hot gas chemistry, and its conversion into more complex molecules (Charnley et al. 1992). Therefore, in all the environments considered here, the conversion between ortho and para forms of formaldehyde by the process considered in this paper is very unlikely. If no other faster conversion mechanism exists in the interstellar medium, the measure of the ortho-to-para ratio can be used as a reliable measure for the temperature of molecule formation.

\section{Conclusion}

In typical gas-phase protostar environments, the probability that a conversion of the total spin of $\mathrm{H}_{2} \mathrm{CO}$ occurs is close to zero.

Although conversion is not impossible, it is far too low for a gaseous phase with non reactive collision mechanism. In fact, other mechanisms mentioned in the introduction involving chemical reaction (proton exchange, etc.) but also the adsorption-desorption process on grains have to be considered as a potential source of nuclear spin conversion. They should therefore be evaluated to get an overall view of the spin conversion rate of $\mathrm{H}_{2} \mathrm{CO}$ in interstellar space.

Acknowledgements. The authors acknowledge Dr. Agnès Perrin for fruitful discussions and recent spectroscopic data on $\mathrm{H}_{2} \mathrm{CO}$. This work is part of the scientific program of the Centre d'Études et de Recherches Lasers et Applications (CERLA), which is supported by the Ministère de la Recherche, the Région Nord-Pas de Calais, and the Fonds Européen de Développement Économique des Régions.

\section{References}

Aikawa, Y., van Zadelhoff, G. J., van Dishoeck, E. F., \& Herbst, E. 2002, A\&A, 386,622

Birnbaum, G. 1967, J. Chem. Phys., 42, 2455

Blake, G. A., Sandell, G., van Dishoeck, E. F., et al. 1995, ApJ, 441, 689

Burkart, M., \& Schramm, B. 2003, J. Mol. Spectrosc., 217, 153

Cacciani, P., Cosléou, J., Herlemont, F., et al. 2006, J. Mol. Struct., 780-781, 277

Cacciani, P., Cosléou, J., Herlemont, F., Khelkhal, M., \& Lecointre, J. 2004, Phys. Rev. A, 69, 032704

Carter, S., \& Handy, N. C. 1996, J. Mol. Spectrosc., 179, 65

Chapovsky, P. L. 1991, Phys. Rev. A, 43, 3624

Chapovsky, P. L. 2001, J. Mol. Struct., 599, 337

Chapovsky, P. L., Cosléou, J., Herlemont, F., Khelkhal, M., \& Legrand, J. 2000, Chem. Phys. Lett., 22, 424

Charnley, S. B., Tielens, A. G. G. M., \& Millar, T. J. 1992, ApJ, 399, L71

Corner, L., Barry, H., \& Hancock, G. 2003, Chem. Phys. Lett., 374, 28

Curl, Jr., R. F., Kasper, J. V. V., \& Pitzer, K. S. 1967, J. Chem. Phys., 46, 3220

Dalgarno, A., Black, J. H., \& Weisheit, J. C. 1973, Astrophys. Lett., 14, 77

Dello Russo, N., Bonev, B., DiSanti, M., et al. 2005, ApJ, 621, 537

Dickens, J., \& Irvine, W. 1999, ApJ, 518, 733

Doty, S. D., Everett, S. E., Shirley, Y. L., Evans, N. J., \& Palotti, M. L. 2005 , MNRAS, 359, 228

Dunning, T. H. Jr. 1989, J. Chem. Phys., 90, 1007

Fabricant, B., Krieger, D., \& Muenter, J. 1977, J. Chem. Phys., 67, 1576

Gauss, J., Ruud, K., \& Helgaker, T. 1996, J. Chem. Phys., 105, 2804

Green, S. 1991, ApJS, 76, 979

Jansen, D. J., van Dishoeck, E. F., \& Black, J. H. 1994, A\&A, 282, 605

Jørgensen, J., Schöier, F., \& van Dishoek, E. 2005, A\&A, 437, 501

Kahane, C., Frerking, M., Langer, W., Encrenaz, P., \& Lucas, R. 1984, A\&A, 137,211

Kendall, R. A., Dunning, T. H. Jr., \& Harrison, R. J. 1992, J. Chem. Phys., 96, 6796

Landau, L. D., \& Lifshitz, E. M. 1981, Quantum Mechanics, 3rd edn. (Pergamon Press, Oxford Edition)

Maret, S., Ceccarelli, C., Caux, E., et al. 2004, A\&A, 416, 577

Markwick, A. J., Millar, T. J., \& Charnley, S. B. 2000, ApJ, 535, 256

Miani, A., \& Tennyson, J. 2004, J. Chem. Phys., 120, 2732

Mumma, M., Weaver, H., \& Larson, H. 1987, A\&A, 187, 419

Müller, H. S. P., Winnewisser, G., Demaison, J., Perrin, A., \& Valentin, A. 2000, J. Mol. Spectrosc., 200, 143

Nerf, Jr., R. 1975, J. Mol. Spectrosc., 58, 451

Neufeld, D. A., Melnick, G. J., \& Harwit, M. 1998, ApJ, 506, L75

Niko van Hulst, F., Ter Meulen, J., \& Dymanus, A. 1987, J. Chem. Phys., 86, 4461

Ohishi, M., Ishikawa, S.-I., Amano, T., et al. 1996, ApJ, 471, L61

Peters, G., \& Schramm, B. 1999, Chem. Phys. Lett., 302, 181

Puzzarini, C., Coriani, S., Rizzo, A., \& Gauss, J. 2005, Chem. Phys. Lett., 409, 118

Raghavachari, K., Trucks, G. W., Pople, J. A., \& Head-Gordon, M. 1989, Chem. Phys. Lett., 157, 479

Schöier, F. L., Ryde, N., \& Olofsson, H. 2002, A\&A, 391, 577

Stanton, J. F., Gauss, J., Watts, J. D., Lauderdale, W. J., \& Bartlett, R. J. 1992 , Int. J. Quantum Chem. Symp., 26, 879

Sun, Z.-D., Takagi, K., \& Matsushima, F. 2005, Science, 310, 1938

Tielens, A. G. G. M., \& Allamandola, L. J. 1987, in ASSL, 134, Interstellar Processes, 397

Tielens, A. G. G. M., \& Hollenbach, D. 1985, ApJ, 291, 722

Watanabe, N., \& Shiraki, T. 2003, ApJ, 588, L121

Woon, D. E., \& Dunning, Jr., T. H. 1995, J. Chem. Phys., 103, 4572 